\title{
Aspects of Collagen Changes in Breast Cancer
}

\section{Claudio Luparello*}

Dipartimento di Scienze e Tecnologie Biologiche, Chimiche e Farmaceutiche (STEBICEF), Università di Palermo, Italy

\begin{abstract}
It is widely acknowledged that the Extracellular Matrix (ECM) of connective tissues plays active roles in numerous biological processes such as cell differentiation, life/death promotion and carcinogenesis. Breast cancer is commonly associated to drastic massive alterations of ECM architecture and composition, especially for what concerns its collagenous component. This mini-review is not aimed to give a comprehensive overview of this complex biological event, but to pick up selected early and recent studies on peculiar features of this field and recapitulate specific aspects related to collagen changes in the affected stroma, such as the re-appearance of OF/LB collagen the accumulation of type $\mathrm{V}$ collagen and the identification of tumour-associated collagen signatures at structural and gene expression levels. In addition, the effects of OF/LB and type $V$ collagens on the phenotype of cultured breast cancer cells, as well as recent data on the active role played on neoplastic cells by collagen fragments originating from prominent tissue turn-over are summarized and discussed.
\end{abstract}

\section{Extracellular Matrix Modifications in Breast Cancer}

A great deal of experimental evidence has demonstrated that the Extracellular Matrix (ECM) is not to be regarded as a mere passive scaffold for connective tissues within organ architecture, but that cellECM interactions may influence a number of biological activities such as cell proliferation, differentiation, biosynthetic ability, polarity and locomotion via a number of structurally-different receptors. The study of the biological complexity of the ECM and its constituents has allowed gathering so far an extensive number of data. Each contributing to the knowledge of not only the dynamic relationships between the different components of connective tissues which regulate cell signalling and gene expression in normal conditions, but also the relevant role played by the ECM components in the onset of a considerable number of diseases.

The metastatic process of carcinomas can be schematized as a series of events that lead to: i) the invasive in growth of cancer cells into the primary site after the dissolution of the sub-epithelial basement membrane. ii) the "intravasation" of the more malignant cell variants into the local vessels after the lysis of the sub-endothelial basement membrane, and, iii) the "extravasation" and eventual colonization of anatomically-distant sites exposing molecular determinants that may allow tumour cell adhesion. One of the key features of the first step of this chain of events, i.e. the colonization of the primary site, is the perturbation of the delicate equilibrium of molecular mechanisms governing host tissue normal physiology and homeostasis. Already in 1982, Nicolson had postulated that the microenvironment present within the host tissues might exert an influence on the behaviour of cancer cells by modulating their phenotype. And in his review articles of 1984 and 1986, once verified the occurrence of morphological alterations of the breast stroma in the pre-neoplastic stage as well as during the invasive growth. van den Hooff had tentatively proposed that signal molecules emanating from the ECM, such as collagens and their peptides, could "contribute to at least one aspect of ultimate malignancy of neoplastic cells" [1-4]. After more than two decades, it is now widely recognized that cells and ECM do exchange "instructive" signals in both directions. In fact, if on one hand tumour- and tumourstimulated host cells acquire the ability to release and accumulate in breast ECM a number of newly synthesized macromolecular components and to regulate their pattern of assembly, on the other hand the new composition and architecture of the ECM can exert a prominent feedback effect on the control of the malignant phenotype of cells scattered in it [5]. The recent utilization of in vitro 3D microengineered cell-ECM systems and hybrid cell-ECM models $[6,7]$ has allowed to obtain information on the drastic effects exerted by changes in micro-environmental composition, topography and stiffness on breast cancer cell attitude to proliferate and migrate into the tissue. Data obtained from animal and human model systems have evidenced changes regarding the amount and distribution of diverse ECM components of tumour-affected breast tissue, such as osteopontin/hyaluronan [8,9], tenascin-C [10], laminin-322 [11], thrombospondin-1 [12], and, most conspicuously, collagens as it will be considered in the following paragraphs.

\section{The Collagen Family: A Concise Overview}

Collagen, a major constituent of connective tissues, is a protein family composed of at least 28 genetically-distinct species; their soluble subunits, tropocollagens, share a triple-helical conformation originating from the coiling of three polypeptides of about 1,000 aminoacids, called $\alpha$-chains. Each of them is characterized by a (Gly$\mathrm{X}-\mathrm{Y})_{\mathrm{n}}$ repeat, with proline and 4-hydroxyproline accounting for a large aliquot of $\mathrm{X}$ and $\mathrm{Y}$, although they display a certain degree of biochemical and functional heterogeneity as well as tissue specificity. The $\alpha$-chain composition of the different collagens can give rise to either homo- or heterotrimeric types with a molecular mass of about $300 \mathrm{kDa}$, that accumulate in the ECM, thereby interacting with other micro-environmental components as well as cell receptors.

In light of the variety in their supra-molecular structures, vertebrate collagens are catalogued in different subgroups. Type I, II, III, V, XI, XXIV and XXVII collagens belong to the category of the fibril-forming collagens, that once processed by removal of their $\mathrm{N}$ - and C-terminal

*Corresponding author: Claudio Luparello, Dipartimento di Scienze e Tecnologie Biologiche, Chimiche e Farmaceutiche (STEBICEF), Università di Palermo, Viale delle Scienze, 90128 Palermo, Italy, Tel: +3909123897405; Fax: +390916577210; E-mail: claudio.luparello@unipa.it

Received April 08, 2013; Accepted May 27, 2013; Published June 10, 2013

Citation: Luparello C (2013) Aspects of Collagen Changes in Breast Cancer. J Carcinogene Mutagene S13: 007. doi:10.4172/2157-2518.S13-007

Copyright: ( 2013 Luparello C. This is an open-access article distributed under the terms of the Creative Commons Attribution License, which permits unrestricted use, distribution, and reproduction in any medium, provided the original author and source are credited. 
telopeptides generate cross-striated fibrils and suprafibrillar aggregates. Type IX, XII, XIV, XVI, XIX, XX, XXI, XXII and XXVI collagens are the fibril-associated collagens with interrupted triple helices (FACITs); they exhibit one or more interruptions, or imperfections, in their triplehelix conformation, and have been shown to associate with ("decorate") fibrillar collagen species, thereby bridging the latters with other ECM constituents. Type XIII, XVII, XXIII and XXV are known as the collagens with transmembrane domain, involved in hemidesmosome and focal adhesion assembly and in ECM compositional changes when their extracellular portion is proteolitically-released from the cell surface. Among the non-fibrillar collagens, hexagonal network-forming collagens (type X), basement membrane collagens (type IV, XV and XVIII, the latter two known as multiplexins), beaded filaments-forming collagens (type VI) and anchoring fibrils-forming collagens (type VII) can be additionally recognized [13].

Different receptors, members of integrin, receptor tyrosine kinase and immunoglobulin-like superfamilies [14], mediate cell adhesion to collagen domains. Among the recognition sites in a-chain primary structures, apart from the "classical" RGD tripeptide [15], the GPO and GFOGER motifs [16], the binding sites in type IV collagen [17], the KGD motif [18] and the NC1 domain [19] have been characterized most extensively.

\section{Ex Vivo Studies on Collagen Composition and Architecture in Breast Cancer ECM}

It is known that the stroma of neoplastic breast undergoes to desmoplastic reaction commonly characterized by the overdeposition of a dense fibrous tissue containing newly synthesized ECM components, and mainly collagen [20]. Desmoplasia leads to the formation of a solid lump, which was traditionally considered as an antagonistic response of the host stroma to the development of the primary tumour; on the other hand, such condensation of the ECM appeared surprisingly ineffective in restraining the metastatic process. Indeed, tumour cells subsist within a tissue context, which provides paracrine communications influencing the behaviour of the disseminated cell types. The more represented non-neoplastic cellular components of tumour stroma include fibroblasts, endothelial and immune cells which, collectively, produce a variety of molecules that accumulate in the tissue microenvironment, such as ECM proteins, enzymes, cytokines and growth factors. Cancer associated fibroblasts (CAF) exert a key effect on the growth promotion of breast tumour cells through a feedback process initiated by a reactive stromal response triggered by neoplastic cells, and followed by a modification of cancer cell responses induced by the modified CAFs via their secretome, distinct to that of normal tissue-associated fibroblasts, which includes stromal cell-derived factor 1 (SDF-1), hepatocyte growth factor (HGF), TGFa1, various metalloproteases and the syndecan SDC-1 [21-23]. CAFs are also involved in the onset of the angiogenic process and in the remodelling of the ECM, being the main cytotype responsible for the over-production of collagen in breast cancer stroma, although a contribution by tumor cell themselves has been reported [24].

Concerning the compositional aspects of the collagen content in the ECM, earlier studies on the Ductal Infiltrating Carcinoma (DIC) of the breast indicated the occurrence of an over-deposition of bundles of type I and III collagens localized at the invasion front of the tumour $[25,26]$. A parallel biochemical characterization of collagen extracted from DIC tissue showed an increment of type $\mathrm{V}$ collagen content up to $10 \%$ of total collagens, whereas it accounts for less than $1 \%$ in normal breast tissue [27]. In light of electron microscopic evidence of the perturbation of type I collagen normal fibrillogenesis in vitro in the presence of increasing concentrations of type $\mathrm{V}$ collagen [28], such tissue over-deposition of type $\mathrm{V}$ collagen might be conceivably linked to a derangement of the physiological organization of type I collagen fibrils and fibres. Due to the formation of mixed components whose accumulation might be instrumental for breast cancer invasion [29].

In addition, the appearance of a new collagen species, which was initially presumed to be a homotrimeric $[\alpha 1(\mathrm{I})]_{3}$ variant of type I, accounting for $40-50 \%$ of total collagens, was also reported $[30,31]$. Noteworthy, when submitted to fibrillogenesis in vitro, this latter collagen formed striated fibrils endowed with a banding pattern different to that of "regular" type I collagen, thus demonstrating to be a stable phenotype and not a product of tumour-dependent dysregulation of the mechanisms of $\alpha_{1}(\mathrm{I})$ chain assembly [32]. Such peculiar modifications of the stromal composition and organization were not observed in other non-tumoural desmoplastic reactions occurring in breast ECM, e.g. fibrocystic mastopathy [33], thereby representing a feature typically linked to the progression of DIC development. To get more into molecular details, the preparations of homotrimeric collagen were submitted to bidimensional electrophoretic separation of its $\alpha$ chain. The data obtained indicated the presence of three distinct polypeptides of about $100 \mathrm{kDa}$, i.e. a unique acidic component with an apparent isoelectric point of 4-5, thereby structurally distinct from the regular $\alpha_{1}(\mathrm{I})$ collagen chain, and two other components migrating as $\alpha_{1}$ (II)and $\alpha_{1}$ (III) collagen chains. Confirmation of the structural diversity of the acidic chain from regular $\alpha_{1}$ (II) and $\alpha_{1}$ (III) chains was obtained by trypsin digestion and sequencing of some resulting peptides [34]. Another peculiar property shown by this collagen type, not shared by regular type I and III collagens, was its strong ability to bind laminin in affinity chromatography experiments; the interaction with laminin required the presence of all three a chain components in native conformation. Noteworthy, collagen of this type was found also in colon carcinoma tissues, as well as in bovine embryonic tissues and human umbilical cord. For all these reasons, the homotrimeric collagen species was termed onco-foetal/laminin-binding (OF/LB) [35].

More recent analyses on the collagenous components of breast cancer biopsy fragments have been focussed on an advanced microscopic approach of morphological modifications in collagen deposition and arrangement aimed to the identification of the socalled Tumour-Associated Collagen Signatures (TACS), and on the evaluation of changes in collagen gene expression.

Multiphoton laser scanning and second harmonic generation microscopy have been used to image collagen organization at high resolution in unfixed, intact, and non-stained breast tumour explants and microarrays [36]. Three different TACS, related to the progression of breast carcinogenesis in mouse models, were described, i.e. TACS1 , which is a limited collagen density localized around small tumour foci. TACS- 2 characterized by stretched collagen fibres tangentiallyoriented along a smooth tumour boundary, and TACS-3 characterized by collagen fibres aligned perpendicularly to an irregular tumour boundary and oriented in the direction of cell invasion [37]. The analysis of a 196 sample-tissue microarray [38] clearly indicated that in human samples TACS-3 may represent a robust and reliable marker associated with disease outcome, especially in those cases such as triple negativebreast cancer where other biomarkers are lacking, thereby allowing to predict breast cancer recurrence and survival in patients. An additional promising aspect of this type of analysis is that it can be performed also on histological slides and therefore potentially incorporated in the clinical practice. In parallel studies, second harmonic generation 
microscopy was also used to monitor collagen fibre density in normoxic and hypoxic areas of breast tumours [39], suggesting a restructuring of collagenous architecture in the hypoxic microenvironment that might influence cancer cell invasive growth. More recently, this imaging technique was employed in a quantitative way, thus investigating collagen structural changes at the molecular scale, evaluating the percentage of abnormal collagen fibrils and developing a numerical parameter, i.e. anisotropic/isotropic (A.I.) region ratio, which estimates the number of areas with aligned or randomly-oriented collagen fibres and allows to differentiate malignant from other breast pathologies [40]. Also, the quantitative evaluation of forward-to-backward second harmonic generation signals have proven useful to differentiate invasive breast cancer and to monitor the progress of collagen changes during breast carcinogenesis [41]. It is worth mentioning that also another technique suited for analysis of large-scale organization of macromolecules, such as small angle $\mathrm{x}$-ray scattering, was successfully employed to the identification of the structural aspects of collagen in breast cancer tissues, allowing discriminating regions of cancer cell invasion and predicting the direction of cancer spreading [42].

Another line of research was addressed to identify collagen gene expression signatures that can represent reliable metastasis-associated biomarkers. The best candidate, which has been the object of a number of recent studies, is COL11A1 encoding for the $\alpha_{1}$ chain of collagen XI. Ellsworth et al. [43] have produced evidence that COL11A1 is upregulated in primary breast cancer compared to the metastatic lymph node, thereby suggesting that this collagen type may contribute to organize permissive ECM enabling metastasizing cells to disseminate. Subsequent studies [44] demonstrated that COL11A1 is synthesized by cancer cell themselves other than tissue fibroblasts, and, interestingly, that its over-expression is paralleled by the up-regulation of COL5A2, encoding for $\alpha_{2}$ chain of collagen type $\mathrm{V}$ whose abnormal deposition in the ECM of DIC was already reported in earlier publications $[27,45]$, as discussed previously. In addition, up-regulation of COL11A1 and COL5A2 has been reported also in colorectal carcinoma tissue [46]. Since both collagens are not expressed in adult human colon but only in foetal gut and intestine, a possible explanation of this finding, which might be extended also to breast cancer for COL11A1, is the carcinogenesis-associated reappearance of an embryologic expression pattern in the affected tissues, as already postulated in the case of $\mathrm{OF} /$ LB collagen. Very recent studies suggest that COL10A1 encoding for the $\alpha_{1}$ chain of collagen type $\mathrm{X}$ might be another gene expression signature for diverse tumoural histotypes, including those of the breast. In particular, immunostaining of the protein product appeared to be selectively positive for breast tumour-associated vasculature compared to normal breast tissue, thereby representing a potential novel biomarker for the diagnosis of breast cancer [47].

\section{In Vitro Studies on the Interactions between Collagens and Breast Cancer Cells}

Since the beginning of the 80 s, researchers utilized collagen as a culture substrate for breast cancer cells to evaluate which could be the "instructive" effect imparted by the film/matrix to the attached cells, being the paper by Leung and Shiu [48] one of the earliest available in PubMed on this field. More recently, collagen-derived peptides representative of the accumulating products of ECM lytic enzyme activity have been also used as supplements to the culture media to monitor their effects on tumour cells.

Concerning the collagen types whose deposition was previously described as abnormal in DIC, i.e. OF/LB and type $\mathrm{V}$, their effects on the phenotype of neoplastic cells of the 8701-BC line, derived from a primary DIC, was the object of a line of investigation. Tumour cells recognized OF/LB collagen as an optimal substrate for adhesion [49] and their attachment determined an increase of proliferation rate compared to uncoated plastic substrate with consequent diminution of the population doubling time from about 47 to 43 hours. An enhanced motile attitude also substantiated by the related reorganization of cytoskeletal actin, which were not observable in cells plated onto regular type I collagen substrate, and an increase in the secretion of metalloprotease-1, -2, -9, and -10 [50-52]. Noteworthy, $\mathrm{OF} / \mathrm{LB}$ collagen substrates were found to induce a faster attachment also of BCS-TC2 colon adenocarcinoma cells, if compared to type I and IV collagen (Luparello and Lizarbe, unpublished results). When modifications of gene expression levels in 8701-BC cells cultured onto OF/LB collagen substrates were analyzed through differential display-PCR. Up regulations of MLL septin-like fusion protein $B$, encoding for a protein involved in cytokinesis and cell division [53], protein phosphatase $2 A$ regulatory subunit $\alpha$-isotype, whose protein product exerts a prominent role in the regulation of cell cycle and mitosis [54], and $h R P B 17$, encoding for one of the subunits of RNA polymerase, were identified [55,56]. Moreover, among different ECM substrates, OF/LB collagen was found to be the most powerful inducer of the release of parathyroid hormone-related peptide (PTHrP), a key element potentially instrumental in promoting carcinogenesis [5759]. Interestingly, either OF/LB or type I collagen were used to fill the wound beds of dermal excisions performed on the back of guinea pigs which were sacrificed after five days. From the histological examination of the OF/LB collagen-containing area, strips of epithelial cells were found to migrate and invade locally the underlying ECM, whilst in the presence of type I collagen the boundary between the epithelium and the granulation tissue remained clearly visible [60]. OF/LB collagen was also found to induce subcutaneous tumours in mice when coinjected with BCS-TC2 colon adenocarcinoma cells (Luparello and Lizarbe, unpublished data). These collective data suggest that in DIC, stromal accumulation of OF/LB collagen, which seems to be ascribed at least in part to tumour cell themselves [61], could be one of the reasons why the dense desmoplastic ECM present in most invasive breast carcinomas [20] appears ultimately unable to impede breast cancer progression. Bundles of this collagen type, in fact, could assemble selected preferential stromal pathways acting as "contact guidance" for a fast dissemination, instead than a drastic obstruction, of attached tumour cells.

Conversely, type $\mathrm{V}$ collagen substrate was a much less efficient substrate for adhesion of 8701-BC breast cancer cells, which appeared to occur through the non-integrinic $67 \mathrm{kDa}$-laminin receptor [62]. The cell subset(s) allowed to adhere onto this collagen substrate showed a decrease of the proliferation rate with a consequent enhancement of population doubling time, from about 47 to 61 hours. Neither locomotion-oriented organization of the cytoskeleton, nor cell motility recorded by time lapse-cinematography, could be observed for cells attached onto type $\mathrm{V}$ collagen $[51,63,64]$. The restraining influence on the proliferative behaviour of DIC cell was maintained also in hybrid type $\mathrm{V} /$ type I collagen substrates, being directly related to the amount of type $\mathrm{V}$ collagen present [28]. When modifications of gene expression levels in 8701-BC cells cultured onto type V collagen substrates were analyzed through differential display-PCR, up-regulation of $m$-calpain, large subunit [65], a neutral $\mathrm{Ca}^{++}$-dependent non-lysosomal enzyme belonging to the cysteine protease family and involved in apoptosis, and down-regulation of the putative transcriptional activator KIAA0309/SRCAP [55], and of protein kinase C-isoform $\delta$, whose 
changes in the expression level are known to be implicated in the onset of post-lactation mammary gland involution in rats [66], were found. Moreover, both 8701-BC and T47-D breast cancer cell lines were proven to be addressed to a death pathway by signals transduced after cell adhesion to type $\mathrm{V}$ collagen substrate. In particular, both exhibited an increase of ethidium bromide-positive nuclei (hallmark of plasma membrane damage), as well as a large percentage of fragmented DNA with an electrophoretic ladder-like pattern (hallmark of apoptotic promotion), and the up-regulation of some apoptosis-related genes [65-68]. Therefore, albeit caution must be exercised in extrapolation of in vitro results to the in vivo situation, type $\mathrm{V}$ collagen seems to be endowed with "tumour-restraining" properties. It might build a natural barrier which inhibits the proliferative/invasive activity of breast cancer cells, subsequently addressing these cells to a death program. Thus, the over-production of this collagen species, which is to be ascribed solely to cells of the mesenchymal compartment, could represent an aspect of the genuine defense reaction of the host tissue to the incoming expansion of the primary tumour.

Recent data have shed some light on different features of the functional interactions between breast cancer cells and collagens, focussing on the role that processing products may play in cell proliferation and invasion, as in the case of type I and VI collagens. Due to the accumulation of proteases around tumour cells in the local breast microenvironment during carcinogenesis, it is indeed conceivable that stable fragments of collagen chains become highly enriched in the stroma compared to full-length molecules.

It is widely acknowledged that type I collagen does not represent a permissive substrate for breast cancer cell proliferation and motility. In fact, apart from a great deal of publications of the past decades on this topic $[50,51,60]$ also more recent reports confirm that breast cancer cells grown into three-dimensional type I collagen gels decrease drastically their proliferation index and that such suppression of proliferation may be ascribed at least in part to down-regulation of cyclin E1, consistent with the inhibition of $G_{1} / S$ phase transition $[69,70]$. On the other hand, the $\mathrm{COOH}$-terminal trimer of procollagen type I, termed $\mathrm{C} 3$, is highly enriched in breast cancer specimens, as well as in bone metastases, due to ECM degradation and remodelling. Noteworthy, this fragment was found responsible of exerting a mitogenic and motogenic effect on both endothelial and MDA-MB231 triple negative breast cancer cells [71]. In addition, C3 triggered the up-modulation of the secretion of vascular endothelial growth factors A and B and exposition of CXCR4 receptor by tumour cells, thereby playing a role in promoting angiogenesis and sustaining cell growth and invasion, as also shown in xenograft models implanted in nude mice $[72,73]$.

Adipocytes are one of the most represented cytotypes in breast tissues and they secrete considerable amount of type VI collagen. Studies performed in rodents and confirmed in human MCF-7 cell line have demonstrated that type VI collagen, whose synthesis by fat cells is up-regulated during mammary tumour progression, stimulates cancer cell proliferation via $\mathrm{NG} 2 /$ chondroitin sulphate proteoglycan receptor, and probably $\alpha_{2} \beta_{1}$ integrin, transducing a signal that stabilizes $\beta$-catenin and up-regulates cyclin D1. Interestingly, the domain that appears to be responsible of such effect is the COOH-terminus of $\alpha_{3}(\mathrm{VI})$ chain, and its isolated cleavage product, also called endotrophin, has been found to co-stimulate TGFa-mediated epithelial-mesenchymal transition of neoplastic cells thereby potentiating cell invasive attitude, and also tissue fibrosis and inflammation via fibroblast activation and chemoattraction of immune cells, respectively $[74,75]$.

\section{Collagen Changes and Breast Cancer Progression: Conclusions and Perspectives}

Considerable progress has been made in the identification of molecular and structural changes of the ECM of neoplastic breast, and in the understanding of the biological consequences of such modifications on the phenotypic expressions of tumour and resident cells [76]. It is now well recognized that mammographic density, which largely reflects variations for collagen, is strongly associated with an increased risk of breast cancer [77]. Tumor-stroma ratio has shown to be a prognostic parameter in breast cancer patients, especially those affected by the triple-negative histotype that lacks definite prognostic biomarkers, independently of other clinico-pathological parameters and systemic therapy [78]. Fibrosis grade was also associated with distant-organ metastasis appearing as more reliable predictive factors for bone metastasis than nodal status [79].

The host environment can indeed play a key prominent role in facilitating the processes of proliferation and motility, which are per se inherent features of cancer cells, thereby profoundly controlling cell invasion and metastatization. The mechanical modification of cell shape and related changes in cytoskeletal architecture imparted in vivo by ECM substrates on cancer cells can play a key role in the switching between phenotypes critical for the carcinogenetic process, such as those related to viability/proliferation, directional motility and differentiation level. In fact, an increase in collagen density massively influences matrix stiffness, which affects the number and nature of binding sites available for neoplastic cell attachment and migration. This, in turn, stimulates metastatic cells to increase contractile force generation and cell spreading, thereby allowing them not only to reorganize their cytoskeletal structure and infiltrate into the dense stroma but also regulate gene expression and differentiation. It is recognized that neoplastic cells undergo to Rho-mediated contraction of the actin-myosin cytoskeleton leading to activation of the downstream signalling pathways including FAK, ERK, and PI3K that promote cell proliferation. On the other hand, the hypoxic microenvironment of large fibrotic foci triggers angiogenesis and lymphangiogenesis via up-regulation of VEGFA and CA9, and potential activation of Ras and Akt signaling [80-83]. Noteworthy, collagen-derived soluble peptides originating from enhanced enzymatic turn-over can also generate signal cascades which drastically reprogram the transcriptional regulation of breast cancer cells via non-attachment-related processes [84].

A great deal of accumulating evidence have unveiled so far only a part of the network of biological interactions occurring between the multitude of stromal components and the cells scattered in the connective tissues. Starting from this point, the ECM is to be perspectively considered as a reservoir full of "natural" components, including collagens, whose potential ability to act as anti-neoplastic agents is still far to be widely exploited, due to the complexity of ECMcell interrelationships. Thus, a more complete understanding of the biological properties of connective tissue components can surely allow cancer biologists to select a new array of molecules that may find several clinically oriented applications, such as coating agents for cell-directed nanoparticles, constituents of chimaeric antibodies directed against tumour cell-specific surface determinants, and components for semisynthetic bio-engineered ECM scaffolds and/or injectable biomaterials. Targeting tumour cell membranes with micro-environmental materials having adequate chemical and physical properties to reverse or attenuate neoplastic aggressiveness may represent a potentially promising approach to develop novel and less harmful anti-cancer treatments. 


\section{Acknowledgments}

This work was supported by a grant of the University of Palermo/Italy (FFR, ex60\%)

\section{References}

1. Nicolson GL (1982) Cancer metastasis. Organ colonization and the cell-surface properties of malignant cells. Biochim Biophys Acta 695: 113-176.

2. van den Hooff A (1983) Connective tissue changes in cancer. Int Rev Connect Tissue Res 10: 395-432.

3. van den Hooff A (1984) The part played by the stroma in carcinogenesis. Perspect Biol Med 27: 498-509.

4. Van den Hooff A (1986) Connective tissue as an active participant in the process of malignant growth. Anticancer Res 6: 775-780.

5. Huang S, Ingber DE (2005) Cell tension, matrix mechanics, and cancer development. Cancer Cell 8: 175-176.

6. Yang CC, Burg KJ (2013) Designing a tunable 3D heterocellular breast cancer tissue test system. J Tissue Eng Regen Med.

7. Kim Y, Othmer HG (2013) A Hybrid Model of Tumor-Stromal Interactions in Breast Cancer. Bull Math Biol .

8. Cook AC, Chambers AF, Turley EA, Tuck AB (2006) Osteopontin induction of hyaluronan synthase 2 expression promotes breast cancer malignancy. J Biol Chem 281: 24381-24389.

9. Götte M, Yip GW (2006) Heparanase, hyaluronan, and CD44 in cancers: a breast carcinoma perspective. Cancer Res 66: 10233-10237.

10. Matei I, Ghajar CM, Lyden D (2011) A TeNaCious foundation for the metastatic niche. Cancer Cell 20: 139-141.

11. Kim BG, An HJ, Kang S, Choi YP, Gao MQ, et al. (2011) Laminin-332-rich tumor microenvironment for tumor invasion in the interface zone of breast cancer. Am J Pathol 178: 373-381.

12. Ioachim E, Damala K, Tsanou E, Briasoulis E, Papadiotis E, et al. (2012) Thrombospondin-1 expression in breast cancer: prognostic significance and association with p53 alterations, tumour angiogenesis and extracellular matrix components. Histol Histopathol 27: 209-216.

13. Grant ME (2007) From collagen chemistry towards cell therapy - a personal journey. Int J Exp Pathol 88: 203-214.

14. Heino J, Käpylä J (2009) Cellular receptors of extracellular matrix molecules. Curr Pharm Des 15: 1309-1317.

15. Elices MJ, Urry LA, Hemler ME (1991) Receptor functions for the integrin VLA3: fibronectin, collagen, and laminin binding are differentially influenced by ArgGly-Asp peptide and by divalent cations. J Cell Biol 112: 169-181.

16. Khew ST, Tong YW (2007) The specific recognition of a cell binding sequence derived from type I collagen by Hep3B and L929 cells. Biomacromolecules 8: 3153-3161.

17. Kanie K, Narita Y, Zhao Y, Kuwabara F, Satake M, et al. (2012) Collagen type IV-specific tripeptides for selective adhesion of endothelial and smooth muscle cells. Biotechnol Bioeng 109: 1808-1816.

18. Nykvist P, Tasanen K, Viitasalo T, Kapyla J, Jokinen J, et al. (2001) The cell adhesion domain of type XVII collagen promotes integrin-mediated cell spreading by a novel mechanism. J Biol Chem 276: 38673-38679.

19. Brassart-Pasco S, Sénéchal K, Thevenard J, Ramont L, Devy J, et al. (2012) Tetrastatin, the NC1 domain of the $\alpha(\mathrm{IV})$ collagen chain: a novel potent antitumor matrikine. PLoS One 7: e29587.

20. Walker RA (2001) The complexities of breast cancer desmoplasia. Breast Cancer Res 3: 143-145.

21. Cirri $P$, Chiarugi $P$ (2011) Cancer associated fibroblasts: the dark side of the coin. Am J Cancer Res 1: 482-497.

22. Orimo A, Weinberg RA (2006) Stromal fibroblasts in cancer: a novel tumorpromoting cell type. Cell Cycle 5: 1597-1601.

23. Aboussekhra A (2011) Role of cancer-associated fibroblasts in breast cancer development and prognosis. Int J Dev Biol 55: 841-849.

24. Al-Adnani MS, Kirrane JA, McGee JO (1975) Inappropriate production of collagen and prolyl hydroxylase by human breast cancer cells in vivo. $\mathrm{Br} J$ Cancer 31: 653-660.

25. Deak SB, Glaug MR, Pierce RA, Bancila E, Amenta P, et al. (1991) Desmoplasia in benign and malignant breast disease is characterized by alterations in leve of mRNAs coding for types I and III procollagen. Matrix 11: 252-258.

26. Kauppila S, Stenbäck F, Risteli J, Jukkola A, Risteli L (1998) Aberrant type and type III collagen gene expression in human breast cancer in vivo. J Patho 186: $262-268$.

27. Luparello C, Rizzo CP, Schillaci R, Pucci-Minafra I (1988) Fractionation of type $\checkmark$ collagen from carcinomatous and dysplasic breast in the presence of alkaline potassium chloride. Anal Biochem 169: 26-32.

28. Pucci-Minafra I, Luparello C (1991) Type V/type I collagen interactions in vitro and growth-inhibitory effect of hybrid substrates on 8701-BC carcinoma cells. J Submicrosc Cytol Pathol 23: 67-74.

29. Ajeti V, Nadiarnykh O, Ponik SM, Keely PJ, Eliceiri KW, et al. (2011) Structura changes in mixed Col I/Col V collagen gels probed by SHG microscopy: implications for probing stromal alterations in human breast cancer. Biomed Opt Express 2: 2307-2316.

30. Pucci Minafra I, Luparello C, Sciarrino S, Tomasino RM, Minafra S (1985) Quantitative determination of collagen types present in the ductal infiltrating carcinoma of human mammary gland. Cell Biol Int Rep 9: 291-296.

31. Luparello C, Pucci-Minafra I (1986) Different behavior of type I and type I-trimer collagen in neutral sodium chloride solutions. Anal Biochem 155: 352-357.

32. Pucci Minafra I, Minafra S, Tomasino RM, Sciarrino S, Tinervia R (1986) Collagen changes in the ductal infiltrating (scirrhous) carcinoma of the human breast. A possible role played by type I trimer collagen on the invasive growth. J Submicrosc Cytol 18: 795-805.

33. Luparello C (1987) Desmoplasia in a non-tumoural disease: determination of collagen content in human mammary dysplasia. Bull Mol Biol Med 12: 49-57.

34. Minafra IP, Andriolo M, Basiricò L, Aquino A, Minafra S, et al. (1995) Onco-fetal/ laminin-binding collagen from colon carcinoma: detection of new sequences. Biochem Biophys Res Commun 207: 852-859.

35. Pucci-Minafra I, Luparello C, Andriolo M, Basiricò L, Aquino A, et al. (1993) A new form of tumor and fetal collagen that binds laminin. Biochemistry 32 7421-7427.

36. Falzon G, Pearson S, Murison R (2008) Analysis of collagen fibre shape changes in breast cancer. Phys Med Biol 53: 6641-6652.

37. Provenzano PP, Eliceiri KW, Campbell JM, Inman DR, White JG, et al. (2006) Collagen reorganization at the tumor-stromal interface facilitates local invasion. BMC Med 4: 38.

38. Conklin MW, Eickhoff JC, Riching KM, Pehlke CA, Eliceiri KW, et al. (2011) Aligned collagen is a prognostic signature for survival in human breast carcinoma. Am J Pathol 178: 1221-1232.

39. Kakkad SM, Solaiyappan M, O'Rourke B, Stasinopoulos I, Ackerstaff E, et al. (2010) Hypoxic tumor microenvironments reduce collagen I fiber density. Neoplasia 12: 608-617.

40. Ambekar R, Lau TY, Walsh M, Bhargava R, Toussaint KC Jr (2012) Quantifying collagen structure in breast biopsies using second-harmonic generation imaging. Biomed Opt Express 3: 2021-2035.

41. Burke K, Tang P, Brown E (2013) Second harmonic generation reveals matrix alterations during breast tumour progression. J Biomed Opt 18: 031106.

42. Sidhu S, Siu KK, Falzon G, Hart SA, Foxe JG, et al. (2009) Mapping structural changes in breast tissue disease using x-ray scattering. Med Phys 36: 3211 3217.

43. Ellsworth RE, Seebach J, Field LA, Heckman C, Kane J, et al. (2009) A gene expression signature that defines breast cancer metastases. Clin Exp Metastasis 26: 205-213.

44. Vargas AC, Reed AE, Waddell N, Lane A, Reid LE, et al. (2012) Gene expression profiling of tumour epithelial and stromal compartments during breast cancer progression. Breast Cancer Res Treat 135: 153-165.

45. Barsky SH, Rao CN, Grotendorst GR, Liotta LA (1982) Increased content of Type $V$ Collagen in desmoplasia of human breast carcinoma. Am J Pathol 108 276-283. 
46. Fischer H, Stenling R, Rubio C, Lindblom A (2001) Colorectal carcinogenesis is associated with stromal expression of COL11A1 and COL5A2. Carcinogenesis 22: $875-878$

47. Chapman KB, Prendes MJ, Sternberg H, Kidd JL, Funk WD, et al. (2012) COL10A1 expression is elevated in diverse solid tumor types and is associated with tumor vasculature. Future Oncol 8: 1031-1040.

48. Leung CK, Shiu RP (1982) Morphological and proliferative characteristics of human breast tumor cells cultured on plastic and in collagen matrix. In Vitro 18: $476-482$

49. Ghersi G, La Fiura AM, Minafra S (1989) Direct adhesion to type I and homotrimer collagens by breast carcinoma and embryonic epithelial cells in culture: a comparative study. Eur J Cell Biol 50: 279-284.

50. Schillaci R, Luparello C, Minafra S (1989) Type I and I-trimer collagens as substrates for breast carcinoma cells in culture. Effect on growth rate, morphological appearance and actin organization. Eur J Cell Biol 48: 135-141.

51. Luparello C, Sheterline P, Pucci-Minafra I, Minafra S (1991) A comparison of spreading and motility behaviour of 8701-BC breast carcinoma cells on type I, I-trimer and type $\mathrm{V}$ collagen substrata. Evidence for a permissive effect of type I-trimer collagen on cell locomotion. J Cell Sci $100: 179-185$.

52. Minafra S, Giambelluca C, Andriolo M, Pucci-Minafra I (1995) Cell-cell and cell-collagen interactions influence gelatinase production by human breastcarcinoma cell line 8701-BC. Int J Cancer 62: 777-783.

53. Surka MC, Tsang CW, Trimble WS (2002) The mammalian septin MSF localizes with microtubules and is required for completion of cytokinesis. Mol Biol Cell 13: 3532-3545.

54. Janssens V, Goris J (2001) Protein phosphatase 2A: a highly regulated family of serine/threonine phosphatases implicated in cell growth and signalling. Biochem J 353: 417-439.

55. Sirchia R, Ciacciofera V, Luparello C (2003) Tumor cell-collagen interactions: Identification and semi-quantitative evaluation of selectively-expressed genes by combination of differential display- and multiplex-PCR. Biol Proced Online 5: 222-227.

56. Sirchia R, Luparello C (2005) Collagen-induced differential expression of an RNA polymerase subunit by breast cancer cells. Biochimie 87: 669-672.

57. Luparello C, Burtis WJ, Raue F, Birch MA, Gallagher JA (1995) Parathyroid hormone-related peptide and 8701-BC breast cancer cell growth and invasion in vitro: evidence for growth-inhibiting and invasion-promoting effects. Mol Cell Endocrinol 111: 225-232.

58. Luparello C, Birch MA, Gallagher JA, Burtis WJ (1997) Clonal heterogeneity of the growth and invasive response of a human breast carcinoma cell line to parathyroid hormone-related peptide fragments. Carcinogenesis 18: 23-29.

59. Luparello C, Schilling T, Cirincione R, Pucci-Minafra I (1999) Extracellular matrix regulation of $\mathrm{PTHrP}$ and $\mathrm{PTH} / \mathrm{PTHrP}$ receptor in a human breast cancer cell line. FEBS Lett 463: 265-269.

60. Pucciminafra I, Luparello C, Aquino A, Basirico L, Minafra S, et al. (1995) Of/Lb collagen promotes chemoinvasion of breast-cancer cells and directs epithelialcell migration into granulation-tissue of experimental dermal wounds. Int $\mathrm{J}$ Oncol 6: 1015-1020.

61. Minafra S, Luparello C, Rallo F, Pucci-Minafra I (1988) Collagen biosynthesis by a breast carcinoma cell strain and biopsy fragments of the primary tumour. Cell Biol Int Rep 12: 895-905.

62. Minafra S, Luparello C, Pucci-Minafra I, Sobel ME, Garbisa S (1992) Adhesion of $8701-\mathrm{BC}$ breast cancer cells to type $\mathrm{V}$ collagen and $67 \mathrm{kDa}$ receptor. J Cell Sci $102: 323-328$.

63. Luparello C, Schillaci R, Pucci-Minafra I, Minafra S (1990) Adhesion, growth and cytoskeletal characteristics of 8701-BC breast carcinoma cells cultured in the presence of type $\mathrm{V}$ collagen. Eur J Cancer 26: 231-240.

64. Luparello C (1994) Adhesion to type $V$ collagen and cloning efficiency in agar of 8701-BC breast cancer cells. Eur J Cancer 30A: 1400-1401.
65. Luparello C, Sirchia R (2011) Type V collagen-induced upregulation of capn2 (large subunit of $\mathrm{m}$-calpain) gene expression and DNA fragmentation in 8701 BC breast cancer cells. Biol Chem 392: 501-504.

66. Luparello C, Sirchia R, Longo A (2013) Type V collagen and protein kinase C $\hat{\imath} \cdot$ down-regulation in 8701-BC breast cancer cells. Mol Carcinog 52: 348-358.

67. Luparello C, David F, Campisi G, Sirchia R (2003) T47-D cells and type V collagen: a model for the study of apoptotic gene expression by breast cancer cells. Biol Chem 384: 965-975.

68. Luparello C, Sirchia R (2005) Type V collagen regulates the expression of apoptotic and stress response genes by breast cancer cells. J Cell Physiol 202: 411-421.

69. Krause S, Maffini MV, Soto AM, Sonnenschein C (2010) The microenvironment determines the breast cancer cells' phenotype: organization of MCF7 cells in 3D cultures. BMC Cancer 10: 263.

70. Wu Y, Guo X, Brandt Y, Hathaway HJ, Hartley RS (2011) Three-dimensional collagen represses cyclin $\mathrm{E} 1$ via $\hat{~}^{2} 1$ integrin in invasive breast cancer cells. Breast Cancer Res Treat 127: 397-406.

71. Palmieri D, Poggi S, Ulivi V, Casartelli G, Manduca P (2003) Pro-collagen $\mathrm{COOH}$-terminal trimer induces directional migration and metalloproteinases in breast cancer cells. J Biol Chem 278: 3639-3647.

72. Palmieri D, Astigiano S, Barbieri O, Ferrari N, Marchisio S, et al. (2008) Procollagen I COOH-terminal fragment induces VEGF-A and CXCR4 expression in breast carcinoma cells. Exp Cell Res 314: 2289-2298.

73. Visigalli D, Palmieri D, Strangio A, Astigiano S, Barbieri O, et al. (2009) The carboxyl terminal trimer of procollagen I induces pro-metastatic changes and vascularization in breast cancer cells xenografts. BMC Cancer 9: 59.

74. lyengar P, Espina V, Williams TW, Lin Y, Berry D, et al. (2005) Adipocytederived collagen $\mathrm{VI}$ affects early mammary tumor progression in vivo demonstrating a critical interaction in the tumor/stroma microenvironment. Clin Invest 115: 1163-1176.

75. Park J, Scherer PE (2012) Adipocyte-derived endotrophin promotes malignan tumor progression. J Clin Invest 122: 4243-4256.

76. McSherry EA, Donatello S, Hopkins AM, McDonnell S (2007) Molecular basis of invasion in breast cancer. Cell Mol Life Sci 64: 3201-3218.

77. Boyd NF, Martin LJ, Yaffe MJ, Minkin S (2011) Mammographic density and breast cancer risk: current understanding and future prospects. Breast Cancer Res 13: 223.

78. de Kruijf EM, van Nes JG, van de Velde CJ, Putter H, Smit VT, et al. (2011) Tumor-stroma ratio in the primary tumor is a prognostic factor in early breas cancer patients, especially in triple-negative carcinoma patients. Breast Cancer Res Treat 125: 687-696.

79. Hasebe T, Imoto S, Yokose T, Ishii G, Iwasaki M, et al. (2008) Histopathologic factors significantly associated with initial organ-specific metastasis by invasive ductal carcinoma of the breast: a prospective study. Hum Pathol 39: 681-693.

80. Keely PJ (2011) Mechanisms by which the extracellular matrix and integrin signaling act to regulate the switch between tumor suppression and tumor promotion. J Mammary Gland Biol Neoplasia 16: 205-219.

81. Van den Eynden GG, Smid M, Van Laere SJ, Colpaert CG, Van der Auwera I, et al. (2008) Gene expression profiles associated with the presence of a fibrotic focus and the growth pattern in lymph node-negative breast cancer. Clin Cancer Res 14: 2944-2952.

82. Paszek MJ, Zahir N, Johnson KR, Lakins JN, Rozenberg GI, et al. (2005) Tensional homeostasis and the malignant phenotype. Cancer Cell 8: 241-254.

83. Kraning-Rush CM, Califano JP, Reinhart-King CA (2012) Cellular traction stresses increase with increasing metastatic potential. PLoS One 7: e32572.

84. Garamszegi N, Garamszegi SP, Shehadeh LA, Scully SP (2009) Extracellular matrix-induced gene expression in human breast cancer cells. Mol Cancer Res 7: 319-329. 